\title{
Session II Summary
}

\author{
Georgia Karagiorgi \\ University of Manchester and Columbia University \\ E-mail: georgia.karagiorgi@manchester.ac.uk and \\ georgia@nevis.columbia.edu
}

\section{Lucio Ludovici}

INFN - Università di Roma Sapienza

E-mail: lucio.ludovicieromal.infn.it

In these proceedings we present a summary of the talks and discussions of Session II, "Oscillation Parameters: Future," at the Neutrino Oscillation Workshop 2016. This session focused on outstanding theoretical questions in neutrino physics as well as experimentally pressing issues which must be overcome in order to solidify our current three-neutrino paradigm and/or establish new physics beyond it.

Neutrino Oscillation Workshop

4 - 11 September, 2016

Otranto (Lecce, Italy) 


\section{Introduction}

The discovery of neutrino oscillation in 2001 substantiated decades of unresolved experimental measurements, signified new physics beyond the Standard Model (SM), and established neutrino oscillation physics as a flourishing field of particle physics research. Today, the most pressing questions in neutrino physics revolve around the determination of the remaining unknown free parameters of the three-neutrino mixing picture. Those include the neutrino mass ordering (whether $\Delta m_{32}^{2}>0 \mathrm{eV}^{2}$ or $\Delta m_{32}^{2}<0 \mathrm{eV}^{2}$ ); the value of the PMNS matrix CP-violating phase, $\delta_{c p}$; and the determination of the octant of the mixing angle $\theta_{23}$. Prospects for determining those parameters with future experimental facilities are discussed in Sec. 2 of this summary. Reaching the necessary precision to determine those parameters and subsequently search for new physics beyond the three-neutrino framework requires improvements in our understanding of neutrino production and neutrino interactions over a wide neutrino energy range. The impact and prospects for improving cross-section modeling uncertainties in particular are summarized in Sec. 3. Such efforts are being pursued with great urgency with current and upcoming dedicated cross-section experiments. What is particularly exciting is that, in the past decade, we have witnessed an exciting revolution in our understanding of neutrino-nucleus scattering, enabled through a wealth of cross-section measurements and new theoretical developments. Reaching a high level of precision in neutrino measurements will enable us to overconstrain the three-neutrino picture, and consequently provide sensitivity to new physics phenomena. Examples are summarized in Secs. 4 and 5. Such phenomena may manifest experimentally in many new ways, such as through effective non-unitarity of the three-neutrino mixing matrix, or different effective oscillation parameters for neutrinos and antineutrinos (a seemingly CPT-violating signature). Of particular interest in recent years have been the experimental hints for sterile neutrinos associated with neutrino masses of $\sim 1 \mathrm{eV}$. The question of whether these additional, sterile neutrinos (and likewise for any other new physics which can be probed through neutrino oscillations) exist is not only of great theoretical consequence, but also of experimental essence. In particular, the extra degrees of freedom introduced by the new physics blurs our yet-incomplete picture of three-neutrino oscillations, and introduces parameter degeneracies and ambiguities in planned experimental searches for $\delta_{C P}$, mass ordering determination, etc. Resolving those ambiguities necessitates independent constraints or measurements of any new physics parameters with dedicated experiments. A summary and prospects for experiments dedicated to sterile neutrino searches is provided in Sec. 4.

\section{Prospects for measurement of oscillation parameters}

The neutrino physics field is steadily marching toward the realization of next-generation experiments which aim to complete our three-neutrino oscillation picture. Notable major international efforts include the long-baseline experiments Hyper-K [1] and DUNE [3], as well as the ORCA and PINGU [5] projects, which are extensions of ongoing underwater and under-ice cherenkov arrays further optimized for atmospheric neutrino oscillation studies. Next-generation underground liquid scintillator detectors including JUNO and RENO-50 [6] will also be necessary to remove the current degeneracy between the known (in particular $\theta_{13}$ ) and unknown oscillation parameters; these experiments will also have a significant sensitivity for neutrino mass hierarchy determination. 
T. Kajita [1] presented the status of ongoing efforts to advance the Hyper-K experimental design toward realization. Hyper-K consists of an upgraded, 1.3 MW neutrino beam from J-PARC paired with two underground water cherenkov detectors, each with $190 \mathrm{kt}$ fiducial mass and 40\% photo-coverage. The Hyper-K collaboration is proposing a staged approach, with the second detector assumed to be ready 6 years after deployment of the first one. A ten-year total run, including this staged approach, is projected to yield a measurement of $\delta_{C P}$ with $\sim 10-25$ degree resolution, depending on the value of $\delta_{C P}$, and compelling sensitivity to $\theta_{23}$ octant determination [2]. Atmospheric neutrino oscillation studies with Hyper-K provide increased sensitivity to the neutrino mass hierarchy, further enhancing Hyper-K's oscillation program. The project is under consideration by the Science Council of Japan, and will be reviewed by the Japanese government in 2017.

D. Stefan [3] presented the status of the DUNE experiment, which employs a 40 kton underground liquid argon detector (LArTPC) as its far detector, together with a 1.2-2.4 MW neutrino beam from Fermilab. After successful beam optimization studies, the DUNE collaboration is now carrying out extensive prototyping and testing to refine the detector design and ensure its technological success. Two design options are under consideration, a single-phase (SP) LArTPC as the reference design, and a dual-phase (DP) LArTPC as an alternative design. A development path has been laid out involving two large-scale prototype detectors, ProtoDUNE SP and DP, which are currently under construction and will be operated soon in a test beam at CERN. These efforts are further supported by the CERN Neutrino Platform [4], which provides an R\&D framework for the international neutrino community. DUNE enables precision oscillation physics and tests of the three-neutrino paradigm, in particular mass hierarchy determination within a few years of data taking, measurement of $\delta_{C P}$ with a few to 10 degrees resolution, determination of the octant of $\theta_{23}$ [2], and tests of unitarity when combined with measurements of $\theta_{13}$ from reactor experiments.

A. Heijboer [5] summarized the ongoing IceCube and KM3NeT projects, both of which have viable and actively pursued low-energy extension programs (PINGU and ORCA, respectively) with significant sensitivity to the neutrino mass hierarchy. ORCA has been adopted as part of KM3NeT, and is partially funded. Construction has began, and completion by 2020 is feasible. PINGU has adopted a new geometry and a shorter timeline for deployment, which can be implemented in 5 years. Both experiments can provide a comparable, $3 \sigma$ determination of the neutrino mass hierarchy in 3-4 years of running, and at higher significance in the case of normal neutrino mass hierarchy and $\sin ^{2} \theta_{23}>0.5$. Both experiments are also sensitive to a wealth of other physics, including sterile neutrinos, non-standard interactions, and PNMS unitarity through $v_{\tau}$ appearance.

A. Garfagnini [6] presented the physics reach and status of next-generation underground reactorbased liquid scintillator detectors: JUNO and RENO-50. JUNO is rapidly progressing toward a final design and start of detector construction. RENO-50 has R\&D funding and work is ongoing to complete the detector design and secure funding for the project, aiming to start data taking immediately after JUNO. Both experiments will constrain $\theta_{13}, \theta_{12}, \Delta m_{21}^{2}$ and $\Delta m_{e e}^{2}$ to $<1 \%$, and enable extended physics programs with supernova burst neutrinos, geoneutrinos, and solar neutrinos.

Dedicated R\&D efforts and facility planning are also essential elements not only for the success of next-generation experiments but also for advancing the neutrino field beyond what nextgeneration experiments can achieve. E. Wildner [7] presented neutrino physics enabled by the planned European Spallation Source (ESS) accelerator, and presented the proposed ESSnuSB project, an intense neutrino superbeam experiment aiming at the discovery of leptonic CP vio- 
lation. ESSnuSB takes advantage of the planned ESS accelerator and proposes the construction of a megaton-scale detector, such as MEMPHYS, at the Garpenberg mine, $\sim 540 \mathrm{~km}$ from the ESS. The projected CP violation discovery potential of ESSnuSB surpasses that of DUNE and Hyper-K. A design report for this project is in preparation.

\section{Impact of neutrino cross-sections on future oscillation experiments}

The challenge for the next generation of neutrino experiments is the observation of small perturbations in the oscillation probabilities, encoding the effects of $\mathrm{CP}$ violation, neutrino mass hierarchy and possibly the presence of additional neutrino states. In order to reach their full physics potential, accelerator-based experiments in particular must control their systematic errors to better than a few percent. The most important sources of systematic errors are the uncertainties in the neutrino nucleus cross-sections where, despite impressive progresses in the recent years, experimental data sets often disagree with each other and we still lack a comprehensive model in the energy range of a few hundred-MeV to a few $\mathrm{GeV}$.

A. Ankowski [8] discussed how this issue has a non negligible impact on the effectiveness of the experimental data to constrain the cross-section systematic in neutrino oscillation experiments. In a nuclear target, neutrino interaction cross-sections are sensitive to multi-nucleon effects and final state interactions for which various models have been proposed. Since the size of these effects is large and there are disagreements between the models, it is important to present experimental measurements in a model-independent way, allowing their comparison with different models, which have to include in their calculation both the primary, nucleon-level cross-sections, as well as nuclear effects and final state interactions. Contrary to electron scattering, the energy of the incoming neutrino can only be inferred from the final state observables, typically the lepton kinematic in charged current quasi-elastic events or the reconstructed calorimetric energy. In the first approach the measured charged lepton energy and scattering angle in quasi-elastic events are used to reconstruct the neutrino energy via a two-body formula assuming that the interaction takes place on a single nucleon at rest. In order to avoid a bias in the neutrino energy reconstruction several effects have to be taken in to account: nuclear spectral functions, collective effects and multi-nucleon excitations, final state interactions between the struck nucleon and the residual nucleus. Moreover, a true quasi-elastic kinematic can be mimicked by single-pion production where the final state pion is reabsorbed in the target nucleus. On the other hand also the calorimetric neutrino energy reconstruction has potential biases depending on the final state event composition due to different energy thresholds and calorimetric responses to protons, neutrons and pions. This requires the knowledge of exclusive cross-sections, an accurate determination of detector responses in test beam experiments and a realistic simulation of nuclear effects, including intra-nuclear cascade [9].

One of the main factors limiting the precision of cross-section measurements is the uncertainty in the neutrino flux. Hadro-production experiments, dedicated to measure the hadron yields on a replica of the actual neutrino beam target, are a crucial ingredient in the determination of the neutrino flux. Nevertheless, measuring neutrino cross-sections to better than 8-10\%, hit the ultimate precision to which the neutrino flux is known. The next generation of $v_{\mu} \rightarrow v_{\mathrm{e}}$ appearance experiments will need to control the systematic uncertainty on the electron neutrino cross-section better than $1-2 \%$ in order to minimize the impact on CPV discovery sensitivity. A. Longhin [10] 
discussed the flux uncertainty in conventional neutrino beams, presenting a novel approach which aims to measure the electron neutrino cross-section at the $1 \%$ level. The idea is to use a secondary hadron beam momentum selected and focused in an instrumented decay tunnel where the $v_{\mathrm{e}}\left(\bar{v}_{\mathrm{e}}\right)$ yield is directly measured by tagging the accompanying positrons(electrons) in $\mathrm{K}_{\mathrm{e} 3}$ decays [11].

\section{Beyond the standard oscillation framework I: sterile neutrinos}

C. Giunti [12] summarized several experimental hints for sterile neutrinos associated with light $(\sim 1 \mathrm{eV})$ neutrino mass states. These hints, which are contributed from both $v_{\mu} \rightarrow v_{e} / \bar{v}_{\mu} \rightarrow$ $\bar{v}_{e}$ appearance and $v_{e} / \bar{v}_{e}$ disappearance searches, are in strong tension with experimental $v_{\mu} / \bar{v}_{\mu}$ disappearance constraints. Nevertheless, when fit together, all experimental datasets support the $(3+1)$ oscillation hypothesis (involving three active and one sterile neutrino) by $>6 \sigma$. The tension is mostly contributed by the MiniBooNE low-energy excess, and thus a "pragmatic approach" can be argued for these fits, whereby the low-energy region of the MiniBooNE data is excluded from consideration, as it is consistent with neither $(3+1)$ nor three-neutrino oscillations. MicroBooNE [13], currently taking data in the Booster Neutrino Beamline, will be crucial for checking the MiniBooNE low-energy anomaly and the consistency of different short-baseline data sets.

A global experimental effort is underway to resolve the outstanding question of what contributes to the LSND, MiniBooNE, Gallium, and Reactor Antineutrino Anomalies. This effort encompasses $v_{e} / \bar{v}_{e}$ disappearance searches with radioactive sources or at very short baselines at reactors, and $v_{\mu} / \bar{v}_{\mu}$ disappearance and $v_{\mu} \rightarrow v_{e} / \bar{v}_{\mu} \rightarrow \bar{v}_{e}$ searches with accelerator beams. Reactorbased disappearance searches may be limited due to the presence of a $5 \mathrm{MeV}$ bump in the reconstructed reactor neutrino energy spectrum consistently observed in recent short- and long-baseline reactor experiments. This has become evident in recent measurements by the NEOS experiment, which nevertheless appears to disfavor sterile neutrino mass splittings below $1 \mathrm{eV}^{2}$. Upcoming experiments including PROSPECT [14] and STEREO [15], both to be deployed within $\sim 10 \mathrm{~m}$ of compact research reactors, are capable of studying antineutrino event rates explicitly in both $L$ and $E$, which will be critical for disentangling potential sterile neutrino oscillation effects from incomplete physics modeling of reactor antineutrino flux spectra. In addition, the Short Baseline Neutrino (SBN) Program [16] will provide 5- $\sigma$ coverage to a large fraction of the currently-allowed $(3+1)$ oscillation parameter space. The SBN program will commence in 2018 and provide explicit tests of $v_{\mu} \rightarrow v_{e}$ appearance and $v_{\mu}$ disappearance.

Should they exist, sterile neutrinos would not only affect long-baseline neutrino oscillation experiments, through their sub-leading effects on oscillations, but would also leave an experimental imprint in beta decay experiments, neutrinoless double-beta decay experiments, solar neutrino measurements, atmospheric neutrino measurements, supernova neutrino and cosmic neutrino searches. Those implications are interesting to explore, but also render it necessary to unambiguously determine whether these new states exist, and, if so, to determine their mass and mixing parameters.

\section{Beyond the standard oscillation framework II: non-standard interactions}

All neutrino oscillation phenomenology observed so far fits in a standard framework of three neutrino families and a unitary lepton mixing matrix. Still, since new particles and new physics 
at an unknown energy scale are required as a SM extension to generate neutrino masses, they could manifest themselves as perturbations of the standard oscillation probabilities. The workshop discussed the effects of new interactions in the neutrino sector, usually known as Non-Standard Interactions (NSI), as well as the effects of a non-unitary mixing matrix. These are in general sub-leading, less than a few percent effects, but as oscillation experiment precision approaches this level we have to be ready both to pin down possible manifestations of new physics and to resolve their potential degeneracy with the determination of the standard oscillation parameters.

New physics affecting neutrino oscillations can be characterized in a wide class of models by four-fermion effective operators, with NSI parameters $\varepsilon_{\alpha \beta}(\alpha, \beta=e, \mu, \tau)$ modifying the standard neutrino production and detection cross-sections. Also the MSW potential for neutrino propagation in matter is changed by the neutral current NSI. It has been pointed out [17] that the existing tension between the mass squared difference from solar neutrino and KamLAND data could be reconciled by considering NSI in neutrino propagation. O. Yasuda discussed the sensitivity to NSI of the future Hyper-Kamiokande experiment in the propagation of atmospheric neutrinos, taking in to account the contributions of all $\varepsilon_{\alpha \beta}$ to the generalized MSW matter potential [18]. It is somehow remarkable that a possible new physics effect, which might be lurking in the low energy (a few $\mathrm{MeV}$ ) solar and KamLAND data, could be definitively observed or refuted at much higher energy by the next generation of atmospheric neutrino experiments.

Within the standard three neutrino mixing scheme the three neutrino flavour and the three mass eigenstates are connected by a $3 \times 3$ unitary mixing matrix $v_{\alpha}=U_{\alpha i} v_{i}$. Additional singlet heavy neutral fermions, like the ones required in many seesaw models, would mix with the flavour neutrinos and, if heavy enough, they will not be produced in low energy experiments. While the total mixing matrix $U^{n x n}$ shall always be unitary, the $3 \times 3$ matrix describing neutrino oscillation would be replaced by a truncated, non-unitary $3 \times 3$ matrix. It can be shown that this can be parametrized by multiplying the standard unitary $3 \times 3$ matrix by a lower triangular $3 \times 3$ matrix [19], containing 6 additional parameters $\alpha_{i j}$. For oscillation experiments involving muon and electron neutrinos there are two additional real parameters, $\alpha_{11}$ and $\alpha_{22}$ and one complex parameter, $\alpha_{21}$, with a new CP phase $\phi$. P. Simoni Pasquini [20] discussed this phenomenology and in particular how the new phase $\phi$ can mimic the CP violation effects of the standard phase $\delta_{C P}$ in long baseline experiments like T2K and T2HK. The degeneracy between the unitary and non-unitary $\mathrm{CP}$ phases could be resolved by complementing the long baseline layout with a source of low energy neutrinos from muon decay at rest ( $\mu$ DAR [21]), at a baseline of $15-23 \mathrm{Km}$.

\section{Conclusions}

The prospects for new discoveries in neutrino physics rest on our ability to reach $1 \%$-level (or better) precision in our measurements of neutrino oscillation parameters. Confirming three-flavor oscillations to this level would enable us to study beyond-Standard Model physics associated with neutrino mass more explicitly within the context of specific models (see, e.g. [22]), while identifying deviations from three-flavor oscillations would signify the discovery of new physics. Reaching this level of precision will require better neutrino sources and improved understanding of neutrino cross-sections on nuclear targets, as near detector constraints are limited in how reliably they can predict far detector signals. Ongoing efforts are directed at addressing some of those limitations, 
aiming to equip next-generation experiments with more robust flux and cross-section models, as well as more precisely measured known oscillation parameters. Within the next decade, it is reasonable to expect a $3 \sigma$ determination of the neutrino mass hierarchy, which is an important parameter for direct neutrino mass and neutrinoless double-beta decay searches. And although some first hints for non-maximal $\theta_{23}$ (for normal hierarchy) and non-zero $\mathrm{CP}$ violation have already surfaced this year [23], new data and significantly increased precision will be necessary to make stronger claims and reach a 5 degree resolution (and increased sensitivity) in $\delta_{C P}$. Finally, the experimental anomalies pointing toward sterile neutrinos seem to be the best evidence we currently have for new physics, and upcoming experiments plan to test these indications with high precision using various independent approaches. Ultimately, whether we confirm or we refute three-flavor oscillations with high significance and precision, we are bound to learn something new about beyond-SM physics.

\section{References}

[1] T. Kajita, these proceedings

[2] S. Vihonen, these proceedings

[3] D. Stefan, these proceedings

[4] P. Sala, these proceedings

[5] A. Heijboer, these proceedings

[6] A. Garfagnini, these proceedings

[7] E. Wildner, these proceedings

[8] A. Ankowski, these proceedings

[9] A. Ankowski et al., Phys. Rev. D 92 (2015) 073014

[10] A. Longhin, these proceedings

[11] A. Longhin, L. Ludovici and F. Terranova, Eur. Phys. J. C75 (2015) 155

[12] C. Giunti, these proceedings

[13] A. Furmanski, these proceedings

[14] K. Gilje, these proceedings

[15] L. Manzanillas, these proceedings

[16] G. Raselli, these proceedings

[17] M. C. Gonzalez-Garcia and M. Maltoni, JHEP 1309 (2013) 152

[18] O. Yasuda, these proceedings

[19] F. J. Escrihuela et al., Phys. Rev. D 92 (2015) 053009

[20] P. Simoni Pasquini, these proceedings

[21] J. Evslin, S. F. Ge and K. Hagiwara, JHEP 1602 (2016) 137

[22] A. Titov, these proceedings

[23] P. Huber, these proceedings 